\title{
Dispersion-Shifted Fiber Polarization Scrambler Based on Faraday Effect
}

\author{
J. Prat, Member, IEEE, and J. Comellas
}

\begin{abstract}
Demonstration of an all-fiber polarization scrambler based on the Faraday effect is carried out. The device has been constructed using dispersion-shifted fiber that has a major tolerance to bends than standard single-mode fiber. Results about the fiber Verdet constant when $1550-\mathrm{nm}$ light is launched are presented. The performance of the constructed device is also shown. Main features are insertion losses as low as $0.4 \mathrm{~dB}$ and scrambling frequency up to $20 \mathrm{kHz}$. Although here we emphasize its application to low-frequency heterodyne detection, the scrambler is applicable to other systems that are polarization dependent. In particular, it would be useful to overcome problems originated by polarization dependent gain in erbium-doped fiber amplified systems.
\end{abstract}

Index Terms- Optical fiber devices, optical fiber measurements, optical fiber polarization.

\section{INTRODUCTION}

$\mathbf{P}$ OLARIZATION effects can severely degrade the performance of many optical systems. Some examples of this are polarization fading in heterodyne detection or polarization dependent gain in long-haul erbium-doped fiber amplifier (EDFA) systems due to anisotropic gain saturation [1]. One possible way to overcome this problem consists of inserting a polarization scrambler in the signal path [2]. Pseudodepolarization of light is achieved, and problems that can arise from the slow random fluctuations of the state of polarization of light (SOP) in fibers can be solved. If we take, as an example, the instantaneous losses in heterodyne detection due to polarization mismatch between the two beams, the problem can be solved by scrambling the SOP of one of them, supposing that the integration time of the receiver is much larger than the scrambling period. This occurs, for example, if heterodyne techniques are used to monitor the carrier wavelengths in wavelength-division-multiplexed (WDM) systems. Using a scrambler, the detected optical signal will be maintained almost constant regardless the SOP variations.

This letter presents the construction and performance measures of a polarization scrambler based on Faraday rotation [3] in optical fiber. A light beam, travelling through a fiber with a magnetic field present, will have its SOP rotated an angle $\theta$ equal to

$$
\theta=V \int B \cdot d l
$$

Manuscript received March 8, 1999; revised April 5, 1999. This work was supported in part by the Spanish CICYT under Project TIC96-1127 and in part by the Generalistat de Catalunya under CIRIT Project 1997SGR409.

The authors are with the Signal Theory and Communications Department, Universitat Politècnica de Catalunya, 08034 Barcelona, Spain.

Publisher Item Identifier S 1041-1135(99)05169-1. where $V[\mathrm{rad} /(\mathrm{m} \cdot \mathrm{T})]$ is called the Verdet constant, and quantifies the strength of the Faraday effect in the medium, $B$ is the magnetic field parallel to the fiber, and $l$ is the length of the light-field interaction. In our case, the fiber is wound and a current carrying conductor is placed around it with toroidal disposition [4]. So, applying Ampere's law, the rotated angle becomes

$$
\theta=\mu V N_{f} N_{c} I
$$

where $N_{f}$ is the number of fiber turns, $N_{c}$ the number of conductor turns and $I$ is the applied electrical current.

Because of the low value of the Verdet constant in silica fibers, it is necessary to have a large number of fiber $\left(N_{f}=\right.$ $1400)$ and conductor $\left(N_{c}=900\right)$ turns. The device constructed consists of dispersion-shifted fiber (DSF) wound on a 90-mmdiameter reel. Using this type of fiber, optical insertion losses are highly reduced and a value as low as $0.4 \mathrm{~dB}$ (including connectors) has been achieved. Using standard single-mode fiber (SMF) [4] losses were about $1 \mathrm{~dB}$. As maximum effect will only be reached if the input light SOP is linear (circularly polarized beams are eigenmodes of a rotator), the random fiber anisotropy has to be minimized. To suppress the effect of the intrinsic and bend induced linear birefringence of the fiber (which could bring the SOP to nonlinear states), it has been twisted at a rate of $120 \mathrm{rad} / \mathrm{m}$ during the winding process. An applied twist induces a high degree of circular birefringence, which biases the total birefringence away from the region of interest.

When frequency rises, the inductance makes the electrical bandwidth of the device to be as low as $800 \mathrm{~Hz}$. As the SOP has to be modulated at frequencies up to $20 \mathrm{kHz}$, we put a series capacitor that compensates the inductance effect. The measured rotator's inductance is $0.1 \mathrm{mH}$. So, maximum response is achieved at $20 \mathrm{kHz}$ with a $680-\mathrm{nF}$ capacitor. Although the electrical resistance of the copper conductor would increase with frequency, its value at $20 \mathrm{kHz}$ is practically equal to its dc value. So we conclude that, using the appropriate capacitor, the device could work at higher frequencies. Limiting this operation at $20 \mathrm{kHz}$ is mainly due to the audio power amplifier used. The complete measurement setup is shown in Fig. 1. In this figure, the current generator incorporates the power amplifier, the capacitor is included in the scrambler block and the polarizer will be used to simulate the polarization dependency of the detector. The different detection instruments will be used in the successive measurements. 


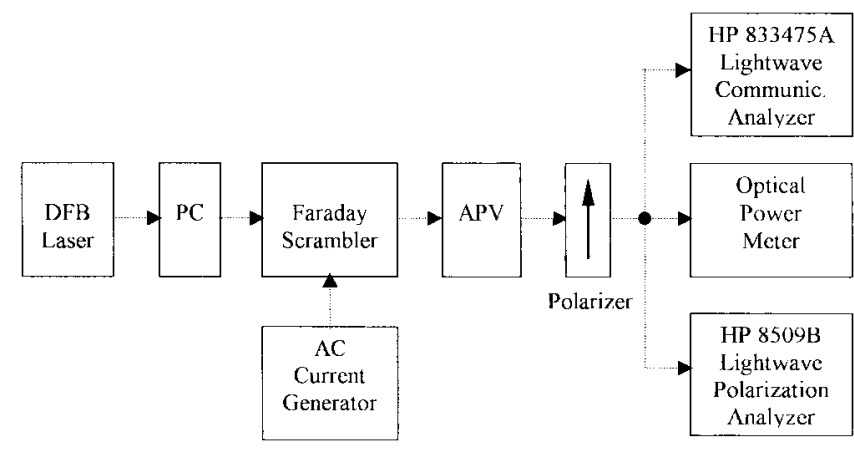

Fig. 1. Experimental setup used to perform the measures. PC: Polarization controller. APV: Automatic polarization variator (this block is only active in the last measurements). Three different detection instruments are used during the measurements.

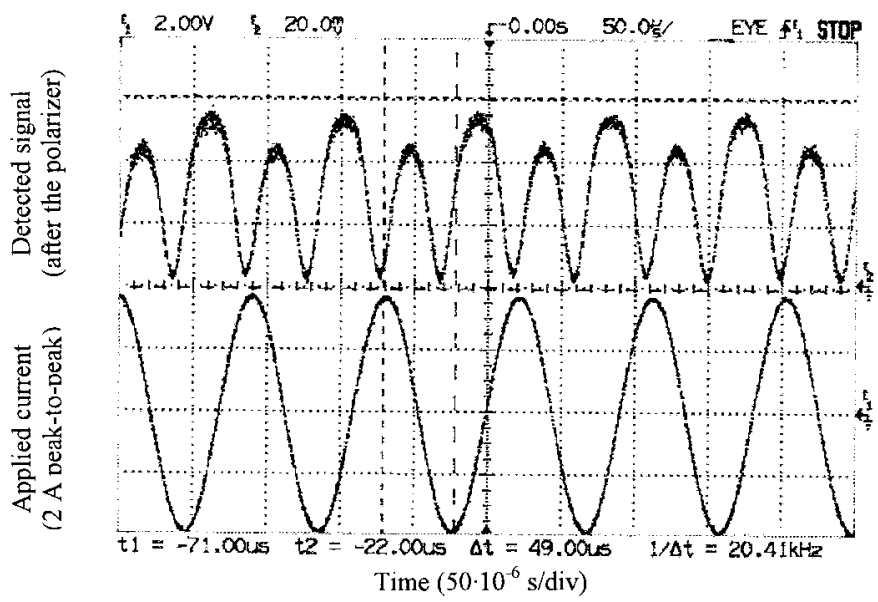

Fig. 2. Scrambler performance. Upper trace is the detected optical power after the polarizer. Lower trace is the applied current.

The performance of the constructed device when a $10-\mathrm{kHz}$ 2-A peak-to-peak sinusoidal current is applied, can be seen in Fig. 2. This graph shows how the detected power beyond the polarizer (see Fig. 1) varies at more than twice the current variation rate, (two maximums appear in every semiperiod of the applied current), so we conclude that about $200^{\circ}$ of rotation are achieved. Maximum detected peaks are not constant (two different values alternate) because the rotation is greater than $\pi$ and less than $2 \pi$. Although in normal operation the input voltage waveform is triangular because its more uniform distribution, sinusoidal current is obtained due to the $L C$ filtering at high frequencies.

As many different measurements were done during the construction and evaluation of the rotator, we extracted an accurate empirical value of the Verdet constant for DSF (for the first time to our knowledge), which is in good agreement with [5]. From measurements taken for wavelengths ranging from 1520 to $1565 \mathrm{~nm}$ we can conclude that the mean value is $V=\left(0.142 \cdot 10^{-28} \pm 0.002\right) \nu^{2} \mathrm{rad} \mathrm{T}^{-1} \mathrm{~m}^{-1}$, where $\nu$ is the light frequency.

The parameter that characterizes the performance of a scrambler is the mean degree of polarization $(\overline{D O P})$, which is defined [6] analogous to the degree of polarization for partially

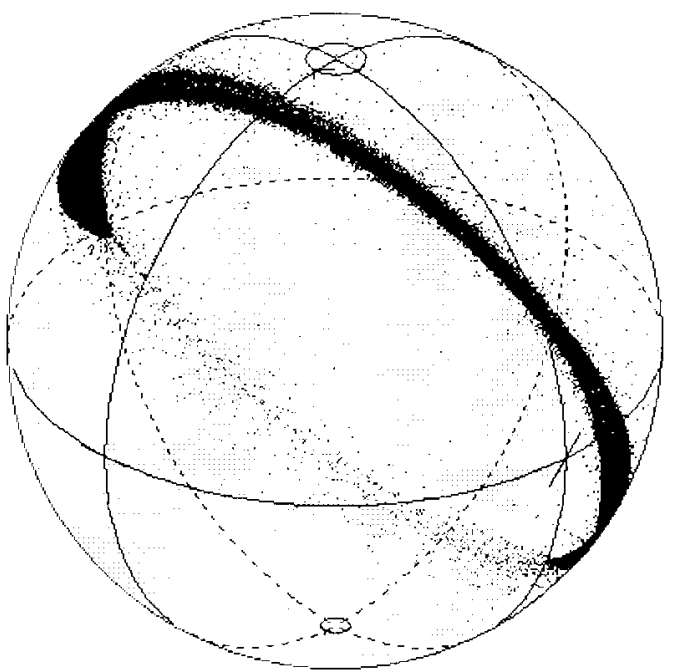

Fig. 3. Scrambler induced SOP variations on the Poincaré sphere. The applied current is a triangular signal with 1-A peak-to-peak and period equal to $25 \mathrm{~ms}$.

polarized light:

$$
\overline{D O P} \equiv \frac{\sqrt{\left\langle S_{1}\right\rangle^{2}+\left\langle S_{2}\right\rangle^{2}+\left\langle S_{3}\right\rangle^{2}}}{S_{0}} \cdot 100
$$

where $\left\langle S_{i}\right\rangle$ are the time averages of the Stokes parameters over a full scrambling period. It is important to remark that, strictly speaking, light is not depolarized, so, its instantaneous DOP will always be near the unity. The technique used here to minimize the $\overline{D O P}$ differs from other previous works [7], [8], which actually depolarize the light by introducing a differential group delay between the orthogonal modes greater than the coherence length. Our device simply makes the light SOP to change very fast, so light appears to be depolarized if the measure time is longer than the scrambling period. This will allow the scrambler to work with very narrow linewidth lasers and high bit-rates.

An ideal scrambler should always maintain the $\overline{D O P}$ close to zero. Considering that some practical nonidealities could appear, mainly due to temperature induced effects, the device's behavior will not be ideal. Nevertheless, $\overline{D O P}$ is always maintained below 30\% during long periods of operation. As shown in [6], this value will be enough for most applications. Fig. 3 shows (measured with the HP8509B Lightwave Polarization Analyzer) a graph of the scrambler performance on the Poincaré sphere. The SOP trajectories are shifted from the equator of the sphere because of the fiber length placed between the scrambler and the HP8509B (see Fig. 1). Trajectories on the Poincaré sphere are slightly varying since there are small environmental fluctuations. As the polarization analyzer has a slow sampling rate, scrambling frequency has had to be reduced, so an actual (not filtered) triangular current is applied to the scrambler. Nevertheless, the current is not enough to achieve a complete circle on the sphere, some points overcome the expected limits because the peak discontinuities of this triangular current shape.

To emulate the highly polarization dependent detection, we performed the experimental arrangement illustrated in Fig. 1. 


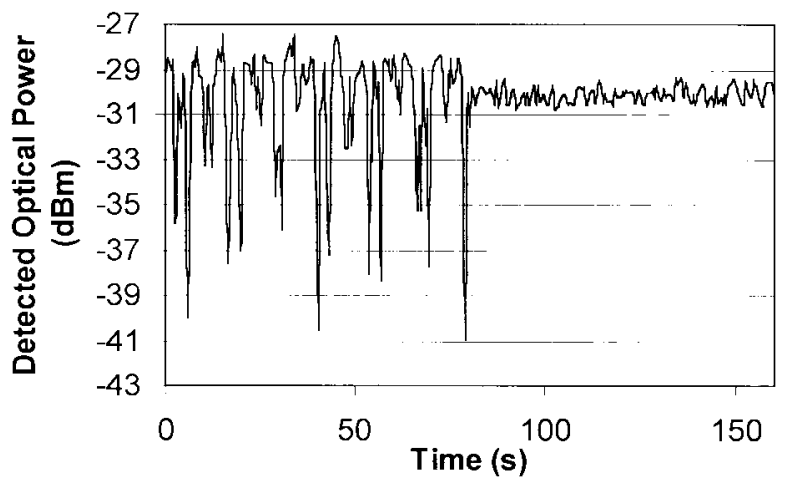

(a)

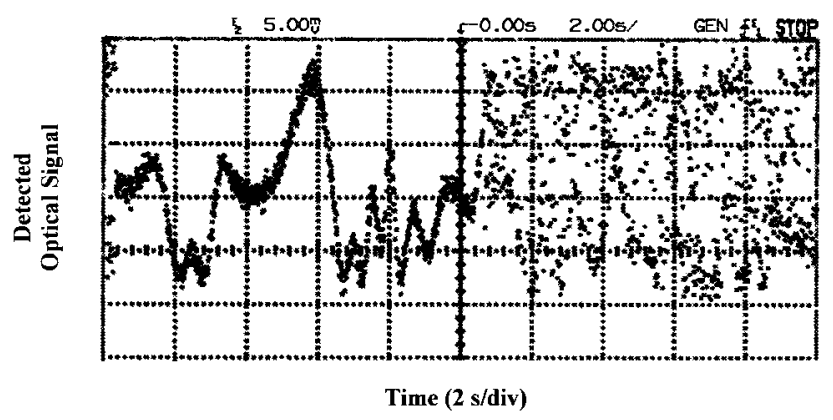

(b)

Fig. 4. Detected power versus time (when APV is active) without and with scrambling. (a) Low-pass filtered. (b) Instantaneous detected signal.

There is a manual polarization controller (PC) at the laser output ensuring that the SOP at the scrambler input is linear, so maximum SOP variation (and then minimum $\overline{D O P}$ ) will be achieved. An automatic polarization variator (APV), which is based on a liquid crystal device, is used to generate great random SOP variations that simulate the SOP fluctuations in a fiber. The resulting signal is directed, through the polarizer, to the optical power meter.

Fig. 4(a) shows the obtained results. We can see that when the scrambler is turned off, there are great changes in the detected power. After about $80 \mathrm{~s}$, when the device is switched on, and because the power meter response is much slower than the scrambling frequency, the variations are highly reduced. Two main conclusions can be extracted from this graph. First, maximum detected power is reduced by an amount of approximately $3 \mathrm{~dB}$. This agrees with expected results because the mean value over a period is exactly half of the maximum. Second, peak-to-peak fluctuations are reduced from 13 to 1.6 $\mathrm{dB}$. This means that the misalignment between the actual scrambled SOP and the ideal mean SOP is about $20^{\circ}$ in the worst case.

Fig. 4(b) shows the instantaneous optical power detected during $50 \mathrm{~s}$ using the HP 83475 Lightwave Communications Analyzer. In the first half of this trace, the scrambler is turned off so great variations in detected power can be observed, but all points follow a continuous trend because the APV induced variations are slow. When the scrambler is active the detected values are scattered and it is not possible to observe this continuous trace. If a low-pass filter were placed after the photodetector, the mean value would be seen [as occurs in Fig 4(a)].

\section{REFERENCES}

[1] E. Lichtman, "Limitations imposed by polarization-dependent gain and loss on all-optical communication systems," J. Lightwave Technol., vol. 13, pp. 906-913, May 1995

[2] V. Letellier, G Bassier, P. Marmier, R. Morin, R. Uhel, and J. Artur, "Polarization scrambling in $5 \mathrm{Gbit} / \mathrm{s} 8100 \mathrm{~km}$ EDFA based system," Electron. Lett., vol. 30, no. 7, pp. 589-590, 1994.

[3] A. H. Rose, S. M. Etzel, and C. M. Wang, "Verdet constant dispersion in annealed optical fiber current sensors," J. Lightwave Technol., vol. 15, pp. 803-807, May 1997.

[4] J. Prat, J. Comellas, and G. Junyent, "Experimental demonstration of an all-fiber endless polarization controller based on Faraday rotation," IEEE Photon. Technol. Lett., vol. 7, pp. 1430-1432, Dec. 1995.

[5] J. L. Cruz, M. V. Andres, and M. A. Hernandez, "Faraday effect in standard optical fibers: Dispersion of the effective Verdet constant," Appl. Opt., vol. 35, pp. 922-927, 1996.

[6] F. Bruyère, O. Audouin, V. Letellier, G Bassier, and P. Marmier, "Demonstration of an optimal polarization scrambler for long-haul optical amplifier systems," IEEE Photon. Technol. Lett., vol. 6, pp. 1153-1155, Sept. 1994.

[7] P. Shen, J. C. Palais, and C. Lin, "Fiber recirculating delay-line tunable depolarizer," Appl. Opt., vol. 37, no. 3, pp. 443-448, 1998.

[8] K. Mochizuki, "Degree of polarization in jointed fibers: The Lyot depolarizer," Appl. Opt., vol. 23, no. 19, pp. 3284-3288, 1984. 